\title{
The Competitive Effects of Variance-based Pricing
}

\author{
Ludwig Dierks and Sven Seuken \\ Department of Informatics, University Zurich \\ dierks@ifi.uzh.ch, seuken@ifi.uzh.ch
}

\begin{abstract}
In many markets, like electricity or cloud computing markets, providers incur large costs for keeping sufficient capacity in reserve to accommodate demand fluctuations of a mostly fixed user base. These costs are significantly affected by the unpredictability of the users' demand. Nevertheless, standard mechanisms charge fixed per-unit prices that do not depend on the variability of the users' demand. In this paper, we study a variance-based pricing rule in a two-provider market setting and perform a game-theoretic analysis of the resulting competitive effects. We show that an innovative provider who employs variance-based pricing can choose a pricing strategy that guarantees himself a higher profit than using fixed per-unit prices for any individually rational response of a provider playing a fixed pricing strategy. We then characterize all equilibria for the setting where both providers use variance-based pricing strategies. We show that, in equilibrium, the providers' profits may increase or decrease, depending on their cost functions. However, social welfare always weakly increases.
\end{abstract}

\section{Introduction}

In most markets with mostly fixed user bases, providers' costs are largely driven by how much buffer capacity they must keep in reserve. This, in turn, depends on the variance of their users' demand. However, the predominant pricing mechanisms employed in practice do not take this effect into account. Instead, prices are typically set on a per-unit basis, such that every user pays the same for the same product.

In electricity markets, for example, a provider has to make long-term supply decisions. But in real-time, supply and demand always have to be perfectly balanced, which requires a costly buffer infrastructure [Cramton, 2017]. If users would always consume (almost) the same amount of energy, this buffer could be far smaller than with widely varying user demands. Nevertheless, users pay simple per MW/h prices.

Next, consider the market for mobile data. Mobile network providers continuously expand their cell tower infrastructure to be able to satisfy their users' bandwidth needs under peak demand [López-Pérez et al., 2009]. However, most end-users pay a fixed per-gigabyte-price, independent of when they consume it or how variable their demand is. ${ }^{1}$

Finally, consider cloud computing markets, where cloud providers must keep buffers of idle capacity in each compute cluster to handle changing resource demands of already running jobs. Handling the variance of cloud users is particularly difficult given that the resource needs of an individual job may vary by a factor of 10 or 100 over time (see Dierks et al. [2019]). In this domain, the mixture of user types (i.e., their average demand variance) significantly affects the providers' need to supply buffer capacity. Nevertheless, most cloud resources are sold for a fixed price per core-hour, without regard to the variability of the users' demand.

Managing Demand via Sophisticated Pricing. Classic approaches for dealing with varying demand include dynamic pricing and congestion-based pricing [Muratori and Rizzoni, 2015; Rong et al., 2018; Truong-Huu and Tham, 2014]. These approaches focus on flattening demand peaks. A big downside is that they make it unpredictable for users whether they can obtain the product at a given price when they need it. This puts providers who serve risk-averse users or users with relatively uniform but inelastic demand at a competitive disadvantage. In some markets, like cloud computing, this effect is so strong that providers never consider dynamic pricing for their primary market offerings [Dierks and Seuken, 2019]. The effect even greatly hinders the adoption of dynamic pricing in more suitable domains, like electricity markets [Joskow and Wolfram, 2012], where customers are instead often only exposed to fixed "time-of-use" tariffs [Urieli and Stone, 2016; Celebi and Fuller, 2012].

Variance-based Pricing. In this paper, we study variancebased pricing, where part of the price the users pay depends on the variance of their demand. Variance-based pricing was recently proposed by Dierks et al. [2019] to reduce costs by improving the demand prediction ability of a monopolistic cloud provider. For a provider, in general markets, variancebased pricing has the advantage that his low-variance users pay lower prices and are thus impacted less by the buffer requirements (which are mainly caused by high-variance users). This is not only fairer, but importantly, it incentivizes users to reduce their variance, which in turn reduces the provider's

\footnotetext{
${ }^{1}$ Even in high-GDP countries, less than $10 \%$ of customers have unlimited data plans [Ericsson, 2017].
} 
costs. In a monopolistic setting, the provider can obviously use variance-based pricing to increase his profits. However, in a competitive market environment, the effects are unclear, because the competitive pricing pressure by other providers may limit what he can achieve with variance-based pricing.

Overview of our Approach. We analyze a duopoly of providers who compete for a continuum of user types. We assume that the two providers consider the long-term effect of their pricing strategies on their profits. Since each provider has to provision enough capacity to guarantee service quality even during demand spikes, the cost per unit of a product depends on the average variance of the users he attracts. Each provider either conservatively employs constant perunit prices or is willing to innovate and employ per-unit prices that linearly depend on each user's variance. We restrict ourself to linear prices here, because their simplicity makes them most plausible and marketable in practice. We show that, as long as a provider's costs are not far larger than the costs of his competitor, unilaterally switching to variance-based pricing can be used to obtain a higher profit for any reasonable constant response of the other provider. We then characterize all equilibria that arise if both providers employ variancebased pricing. We also show that, as long as providers are not symmetric in their cost functions, the profits of both providers often increase, as they can attract user types which they can better serve due to their respective cost functions. Finally, we show that the welfare may decrease if only one provider employs variance-based pricing, but that it at least weakly increases if both employ variance-based pricing.

Related Work. Variance-based pricing is a type of price discrimination [Varian, 1989; Mussa and Rosen, 1978]. However, in contrast to the classic price discrimination settings [Moorthy, 1984; Blattberg and Wisniewski, 1989; Gallego et al., 2006], variance-based pricing does not discriminate based on user preferences. Furthermore, in our model, there is neither a fixed marginal cost of supplying a given user nor do costs depend solely on the number of supplied products. As long as neither provider charges for variance, they cannot price discriminate at all and the problem becomes similar to a Bertrand competition [Baye and Kovenock, 2017]. In order to isolate the competitive effects caused by variance-based pricing, we assume that both providers offer the same product; thus, product differentiation (e.g., [Feng et al., 2013]) does not take place.

\section{Preliminaries}

We consider a market setting with two providers and a continuum of users to which the providers want to sell their products. We study a simple two-period model: in the first period, the providers choose their pricing strategies; in the second period, the users choose a provider to buy the product from.

\subsection{Formal Model}

Each user is associated with a real-valued type $t \in\left[0, t_{\max }\right]$. We keep this type general, but in practice it can be assumed to encode the variance of a user's demand. To keep the notation simple, we normalize each user's expected demand to 1 . For a randomly chosen user, her type is distributed as a continuous random variable with pdf $f(t)$ and cdf $F(t)$. We do not model fluctuations in the mixture of user types over time.

Each provider's strategy space consists of his choice of price function $\rho_{i}$. We restrict $\rho_{i}=\left(p_{i}^{f}, p_{i}^{\ell}\right)$ to a fixed price $p_{i}^{f}$ per unit of the product (independent of the user type) and a second linearly type-based charge $p_{i}^{\ell} t$ per unit of the product. $^{2}$ The overall payment per unit of the product for a user with type $t$ is given by $\rho_{i}(t)=p_{i}^{f}+p_{i}^{\ell} t$. Going forward, we refer to providers that are willing to choose any $p_{i}^{f}, p_{i}^{\ell} \in \mathbb{R}$ as innovative and those who do not adopt the new pricing scheme and restrict themselves to $p_{i}^{\ell}=0$ as conservative.

Given the provider's price functions, and depending on a user's own type, each user chooses to obtain the product either from provider 1 or 2 . We denote a strategy profile for all user types by $\sigma(t):\left[0, t_{\max }\right] \rightarrow\{1,2\}$. In this paper, we do not model the users' values for product consumption. ${ }^{3}$ Consequently, the users' utility function is simply equal to their negative payments. Throughout the paper, given price functions $\left(\rho_{1}, \rho_{2}\right)$, we assume that users only play utilitymaximizing (i.e., payment minimizing) user strategy profiles. Formally, we assume that $\sigma(t)=\operatorname{argmin}_{i}\left\{\rho_{i}(t)\right\}$ for all $t$.

As we will see, it is often in a provider's best interest to play essentially the same strategy as their opponent, which makes tie-breaking rules for the user sub-game very important. To avoid that tie breaking for $\rho_{1}=\rho_{2}$ gives rise to arbitrary user strategy profiles that would not arise in practice, we restrict user strategy profiles to those at least one provider can enforce by deviating from $\left(\rho_{1}, \rho_{2}\right)$ at only an infinitesimal profit loss. Formally, we say that a user strategy profile $\sigma$ is enforceable at $\left(\rho_{1}, \rho_{2}\right)$ if for one provider $i \in\{1,2\}$ there exists a sequence of pricing functions $\left(\rho_{i}^{k}\right)_{k=1}^{\infty}$ such that $\sigma$ is the limit of the user strategy profiles that are uniquely (up to a null set) utility maximizing for each $\left(\rho_{i}^{k}, \rho_{-i}\right)$. With linearly type-based prices, this limits the user strategy profiles to any form where all users with type greater than some cutoff point $\hat{t}$ join one provider $i$ and those with lower type join the other provider. To denote this, we also write $\sigma=[0, \hat{t}]_{\rightarrow i}$ or $\sigma=\left[\hat{t}, t_{\max }\right]_{\rightarrow i}$. Note that this allows for the unique representation of any $\sigma$, as all other users choose the other provider. Further, we denote by $\mu(a, b)$ the average type of all users with type in $[a, b]$, i.e., $\mu(a, b)=\frac{\int_{a}^{b} f(t) t d t}{F(b)-F(a)}$.

A provider's costs for serving a given user do not only depend on how many units of his product he sells to that user, but also on how many units the provider has to supply for all users. Consequently, a provider's cost function $c_{i}(\sigma)$ is a function of the whole user strategy profile $\sigma$ and cannot be separated into independent costs for individual users. We assume that $c_{i}(\sigma)$ is strictly increasing in the expected type of a randomly drawn user who joins provider $i$ 's market under $\sigma$.

\footnotetext{
${ }^{2}$ As supply decisions (and therefore costs) do not depend on a user's true type but on the type the provider predicts for a user, we assume that providers know each realized user's type. In practice, user bases are often mostly fixed, such that a provider can learn each user's type over time.

${ }^{3}$ In the markets we study, essentially every user is served by some provider, as costs are very low compared to most users' values and competition ensures that prices are close to costs.
} 
Overloading notation, we also write $c_{i}(a, b)$ for provider $i$ 's cost if all users in $[a, b]$ (and no other users) choose him.

In many applications, splitting a given population of users between two identical providers causes higher overall costs than if one provider would obtain all users, as that one provider could always provision for both sub-populations separately. We call such cost functions that are convex in relation to splitting the market split-convex, i.e. $c_{i}()$ is splitconvex if for all $\hat{t} \in\left[0, t_{\text {max }}\right]$ it holds that

$$
F(\hat{t}) c_{1}(0, \hat{t})+(1-F(\hat{t})) c_{1}\left(\hat{t}, t_{\max }\right) \geq c_{1}\left(0, t_{\max }\right) .
$$

If the inequality is strict for all $\hat{t} \in\left[0, t_{\max }\right]$, we call the cost function strictly split-convex.

A provider's utility is his expected profit $\pi_{j}$ per customer in a population, which is given by

$$
\pi_{j}\left(\rho_{1}, \rho_{2}, \sigma\right)=\int_{t: \sigma(t)=j}\left(\rho_{j}(t)-c_{j}(\sigma)\right) f(t) d t
$$

Given this model, we call a strategy $\rho_{i}$ for provider $i \in$ $\{1,2\}$ an individually rational response to any given strategy $\rho_{-i}$ of the other provider if there exists an enforceable utility-maximizing user strategy profile $\sigma$ that gives provider $i$ weakly positive profit, i.e., $\pi_{i}\left(\rho_{i}, \rho_{-i}, \sigma\right) \geq 0$.

\subsection{Equilibrium Concept}

We use the following equilibrium concept for our analysis.

Definition 1. A tuple $\left(\rho_{1}, \rho_{2}, \sigma\right)$ is a Bayes-Nash equilibrium $(B N E)$ if $\sigma$ is utility maximizing for $\left(\rho_{1}, \rho_{2}\right)$ and, for $i \in\{1,2\}$, there exist no $\hat{\rho}_{i} \neq \rho_{i}$ and $\hat{\sigma}$ such that $\hat{\sigma}$ is utility maximizing for $\left(\hat{\rho}_{i}, \rho_{-i}\right)$ and

$$
\pi_{i}\left(\hat{\rho}_{i}, \rho_{-i}, \hat{\sigma}\right)>\pi_{i}\left(\rho_{i}, \rho_{-i}, \sigma\right) .
$$

Note that when $\rho_{1}=\rho_{2}$, users are indifferent between all user strategy profiles, but our equilibrium definition mandates that tie breaking is done in such a way that no provider has an incentive to deviate infinitesimally only to secure himself a different user strategy profile. In practice, a combination of external factors and bounded rationality imply that providers do not move their prices to a tie or even infinitesimally close to each other, at best achieving $\epsilon-$ BNEs. Essentially, price differentiations that are too small are not marketable.

\section{Profit Analysis with Conservative Providers}

We first analyze the case where both providers are conservative, i.e., restricted to $p_{1}^{\ell}=p_{2}^{\ell}=0$. Since they cannot split the market through pricing differences, the resulting game is similar to a classic Bertrand competition. Thus, if the providers' costs for the whole population are symmetric, they cannot extract any profit, while for non-symmetric providers, the provider with lower costs for serving the whole market can potentially extract the cost difference as a profit.

Proposition 1. Let both providers be conservative, i.e., $p_{1}^{\ell}=$ $p_{2}^{\ell}=0$. W.l.o.g. assume $c_{1}\left(0, t_{\max }\right) \leq c_{2}\left(0, t_{\max }\right)$. Then in any $\operatorname{BNE}\left(\rho_{1}, \rho_{2}, \sigma\right)$ the following holds:

$$
p_{1}^{f}=p_{2}^{f} \in\left[c_{1}\left(0, t_{\max }\right), c_{2}\left(0, t_{\max }\right)\right]
$$

and

$$
\begin{aligned}
& \pi_{1}\left(\rho_{1}, \rho_{2}, \sigma\right)=p_{1}^{f}-c_{1}\left(0, t_{\max }\right) \\
& \pi_{2}\left(\rho_{1}, \rho_{2}, \sigma\right)=0
\end{aligned}
$$

Proof. Note that any tuple $\left(\rho_{1}, \rho_{2}, \sigma\right)$ with $p_{1}^{f}=p_{2}^{f} \in$ $\left[c_{1}\left(0, t_{\max }\right), c_{2}\left(0, t_{\max }\right)\right]$ and $\sigma=\left[0, t_{\max }\right] \rightarrow 1$ is a BNE as neither provider has an advantageous deviation. All users already choose provider 1 , so decreasing his price only reduces his profit, while any price increase makes him lose all users. Since all users choose him, his profit is simply $\pi_{1}\left(\rho_{1}, \rho_{2}, \sigma\right)=p_{1}^{f}-c_{1}\left(0, t_{\max }\right)$. Any lower price for provider 2 on the other hand would lead to all users choosing him, but he would make a negative profit per user. Increasing his price would have no effect on his profit, as no users choose him. Without users, he trivially makes zero profit. As a special case, when $c_{1}\left(0, t_{\max }\right)=c_{2}\left(0, t_{\max }\right)$, then, by the same argument, $p_{1}^{f}=p_{2}^{f}=c_{2}\left(0, t_{\max }\right)$ with $\sigma=\left[0, t_{\max }\right]_{\rightarrow 2}$ is an additional BNE, with both providers obtaining zero profit.

We now show that these are the only BNEs, by showing that any other "potential BNE" leads to a contradiction. First, note that when $p_{1}^{f}<p_{2}^{f}$, every user strictly prefers provider 1. But there always exists a $\hat{p}_{1}^{f}$ with $p_{1}^{f}<\hat{p}_{1}^{f}<p_{2}^{f}$ for which every user still strictly prefers provider 1 , but with a higher payment. Therefore, $p_{1}^{f}=p_{2}^{f}$ has to hold in equilibrium. If $p_{1}^{f}=p_{2}^{f}<c_{1}\left(0, t_{\max }\right)$, provider 1 would make a loss for every user. On the other hand, if $p_{1}^{f}=p_{2}^{f}>c_{2}\left(0, t_{\max }\right)$, then for any $\sigma=\left[0, t_{\max }\right]_{\rightarrow i}$, the other provider $-i$ would have an advantageous deviation in any $\hat{p}_{-i}^{f}$ with $c_{2}\left(0, t_{\max }\right)<\hat{p}_{-i}^{f}<$ $p_{1}^{f}=p_{2}^{f}$. Taken together, this means that there can be no other BNE than those characterized by the proposition.

Going forward, we denote BNEs with two conservative providers as constant BNEs. Note that, while all $p_{1}^{f}=$ $p_{2}^{f} \in\left[c_{1}\left(0, t_{\max }\right), c_{2}\left(0, t_{\max }\right)\right]$ are equilibrium prices, the only reason why any $p_{1}^{f}=p_{2}^{f}<c_{2}\left(0, t_{\max }\right)$ does not lead to a loss for provider 2 is because he obtains no users. This makes $p_{1}^{f}=c_{2}\left(0, t_{\max }\right)$ the only non-pathological BNE.

\section{Profit Analysis with Innovative Providers}

In this section, we analyze the profit when one or both providers are innovative. As a first step, we show that, given a strategy of provider 2, provider 1's profit is upper bounded by the profit he could achieve if he would play the same strategy as provider 2 (and if he could select the utility-maximizing user strategy profile that is most favorable to him).

Proposition 2. Assume provider 2 plays strategy $\rho_{2}=$ $\left(p_{2}^{f}, p_{2}^{\ell}\right)$. Then, for all $\rho_{1} \neq \rho_{2}, \rho_{1}^{\prime}=\rho_{2}$, and all $\sigma$ that are utility maximizing for $\rho_{1}, \rho_{2}$, it holds that $\pi_{1}\left(\rho_{1}, \rho_{2}, \sigma\right)=0$ or $\pi_{1}\left(\rho_{1}, \rho_{2}, \sigma\right)<\pi_{1}\left(\rho_{1}^{\prime}, \rho_{2}, \sigma\right)$.

Proof. First note that for any $\rho_{1} \neq \rho_{2}$, by the linearity of the price function, there exists a unique $\hat{t}$ at which payments in both markets are the same, i.e., $p_{1}^{f}+\hat{t} p_{1}^{\ell}=p_{2}^{f}+\hat{t} p_{2}^{\ell}$ (note that $\hat{t}$ can be outside $\left.\left[0, t_{\max }\right]\right)$. For any utility-maximizing $\sigma$, users with type below $\hat{t}$ choose one provider and those above the other. Now consider some $\rho_{1} \neq \rho_{2}, \pi_{1}\left(\rho_{1}, \rho_{2}, \sigma\right)>0$ with $\hat{t}$ being the corresponding cutoff. If $p_{1}^{f} \leq p_{2}^{f}, p_{1}^{\ell} \geq$ $p_{2}^{\ell}$, then any utility-maximizing user strategy profile is of the 
form $\sigma=[0, \bar{t}]_{\rightarrow 1}$ for $\bar{t}=\min \left(\hat{t}, t_{\max }\right)$ and it holds that

$$
\begin{aligned}
& \pi_{1}\left(\rho_{1}, \rho_{2}, \sigma\right)=\int_{0}^{\bar{t}} f(t)\left(p_{1}^{f}+t p_{1}^{\ell}-c_{1}(0, \bar{t})\right) d t \\
= & \int_{0}^{\bar{t}} f(t)\left(p_{2}^{f}+\hat{t} p_{2}^{\ell}-\hat{t} p_{1}^{\ell}+t p_{1}^{\ell}-c_{1}(0, \bar{t})\right) d t \\
< & \int_{0}^{\bar{t}} f(t)\left(p_{2}^{f}+\hat{t} p_{2}^{\ell}-c_{1}(0, \bar{t})\right) d t \\
= & \pi_{1}\left(\rho_{2}, \rho_{2}, \sigma\right) .
\end{aligned}
$$

The proof works analogously for $p_{1}^{f} \geq p_{2}^{f}, p_{1}^{\ell} \leq p_{2}^{\ell}$.

It directly follows that in all BNEs, both providers play the same strategy, even if one provider is conservative.

Corollary 1. In all BNEs it holds that $\rho_{1}=\rho_{2}$.

We now separately consider the cases where either only one or both providers are willing to employ linear pricing.

\subsection{One Provider is Innovative}

Corollary 1 suggests that only one provider being innovative precludes the existence of a BNE. In the following theorem we show that this is indeed true, unless both providers' cost functions are "very close" to each other.

Theorem 1. Let provider 2 be conservative, i.e., $p_{2}^{\ell}=0$, and provider 1 be innovative. Then there exists a BNE if and only if there exists no $\hat{t} \in\left[0, t_{\max }\right]$ with

$$
\begin{aligned}
& c_{1}\left(0, t_{\max }\right)-F(\hat{t}) c_{1}(0, \hat{t}) \\
\geq & (1-F(\hat{t}))\left(c_{1}\left(0, t_{\max }\right)-c_{2}\left(0, t_{\max }\right)\right) .
\end{aligned}
$$

If a BNE exists then it is equal to a constant BNE.

Proof. By Corollary 1, in all BNEs, both providers use constant pricing functions and for any constant pricing functions that are not part of a constant BNE there exist constant deviations. By Proposition 2, any deviation of provider 1 from a constant BNE is worse than him freely choosing the user strategy profile and not changing his pricing function. He can enforce any user strategy profile where he only obtains users with types lower than some $\hat{t}$. Thus, if $\left(\rho_{1}, \rho_{2},\left[0, t_{\max }\right]_{\rightarrow 1}\right)$ is a constant BNE, then it is still a BNE when provider 1 is innovative as long as there exists no $\hat{t}<t_{\max }$ such that $[0, \hat{t}]_{\rightarrow 1}$ leads to a higher profit than taking the whole market, i.e.,

$$
\begin{aligned}
& c_{1}\left(0, t_{\max }\right)-F(\hat{t}) c_{1}(0, \hat{t}) \\
< & (1-F(\hat{t}))\left(c_{1}\left(0, t_{\max }\right)-c_{2}\left(0, t_{\max }\right)\right) .
\end{aligned}
$$

Otherwise $\left[0, t_{\max }\right]_{\rightarrow 1}$ cannot be a BNE user strategy profile.

Finally, for all $\left(\rho_{1}, \rho_{2},[0, \hat{t}]_{\rightarrow 1}\right)$ with $\hat{t}<t_{\max }$, the users that choose provider 2 have a higher average variance than the average variance of all users. Consequently, $c_{2}\left(0, t_{\max }\right)<$ $c_{2}\left(\hat{t}, t_{\max }\right)$. As prices are constant, provider 2 either makes negative profit (and deviates) or he increases his profit by slightly decreasing his price and taking the whole market.

Theorem 1 means that providers usually have an incentive to become innovative if their competition is conservative. However, becoming innovative and myopically optimizing profit can lead to non-existence of a BNE. In this case, an innovative provider should consider the additional market power he has due to his larger strategy space when choosing his strategy. Specifically, he should take a (myopically) nonoptimal action to restrict his competitor's rational responses. We now show that, if provider 1 does so, he can guarantee himself strictly positive payoff whenever there is an interval of users for which his costs are lower than provider 2's costs for all users (even though this does not constitute a BNE).

Theorem 2. Let provider 2 be conservative, i.e., $p_{2}^{\ell}=0$. If there exists $0<\bar{t}<t_{\max }$ with

$$
\begin{aligned}
c_{1}(0, \bar{t}) & <c_{2}\left(0, t_{\max }\right) \\
\text { and } & c_{1}(0, \bar{t})<c_{1}\left(0, t_{\max }\right)<2 c_{1}(0, \bar{t}),
\end{aligned}
$$

then there exists a strategy $\rho_{1}$ with $p_{1}^{\ell}>0$ that guarantees provider 1 a non-negative payoff for any $p_{2}^{f}$ and a strictly positive payoff for any individually rational response of provider 2.

Proof. Note that for any $p_{1}^{f}<c_{2}\left(0, t_{\max }\right), p_{1}^{\ell}>0$, provider 2 can only achieve a positive profit if he plays $p_{2}^{f}=p_{1}^{f}+\hat{t} p_{1}^{\ell}$ for some $\hat{t}>0$. To guarantee a positive payoff for provider 1 with such a $p_{1}^{f}$ it therefore suffices that

$$
\pi_{1}\left(\rho_{1}, \rho_{2}, \hat{t}\right)=\int_{0}^{\hat{t}} f(t)\left(p_{1}^{f}+t p_{1}^{\ell}-c_{1}(0, \hat{t})\right) d t>0
$$

for all $\hat{t}$. For $0<\bar{t}<t_{\max }$ with $c_{1}(0, \bar{t})<c_{2}\left(0, t_{\max }\right)$ and

$$
c_{1}(0, \bar{t})<c_{1}\left(0, t_{\max }\right)<2 c_{1}(0, \bar{t})
$$

choose $\rho_{1}$ such that $p_{1}^{f}=c_{1}(0, \bar{t})$ and $p_{1}^{\ell}=\frac{c_{1}(0, \bar{t})}{\int_{0}^{t} f(t) t d t}$. Then it holds for $\hat{t} \leq \bar{t}$ :

$$
\begin{aligned}
\pi_{1}\left(\rho_{1}, \rho_{2}, \hat{t}\right) & =\int_{0}^{\hat{t}} f(t)\left(p_{1}^{f}+t p_{1}^{\ell}-c_{1}(0, \hat{t})\right) d t \\
& >\int_{0}^{\hat{t}} f(t)\left(p_{1}^{f}+t p_{1}^{\ell}-c_{1}(0, \hat{t})\right) d t \\
& =\int_{0}^{\hat{t}} f(t)\left(t p_{1}^{\ell}\right) d t \\
& >0
\end{aligned}
$$

For $\hat{t}>\bar{t}$ it holds that

$$
\begin{aligned}
& \pi_{1}\left(\rho_{1}, \rho_{2}, \hat{t}\right) \\
= & \int_{0}^{\hat{t}} f(t)\left(p_{1}^{f}+t p_{1}^{\ell}-c_{1}(0, \hat{t})\right) d t \\
= & \int_{0}^{\hat{t}} f(t)\left(t \frac{c_{1}(0, \bar{t})}{\int_{0}^{\bar{t}} f(t) t d t}-c_{1}(0, \hat{t})-c_{1}(0, \bar{t})\right) d t \\
> & \int_{0}^{\hat{t}} f(t)\left(c_{1}(0, \bar{t})-c_{1}(0, \hat{t})-c_{1}(0, \bar{t})\right) d t \\
= & \int_{0}^{\hat{t}} f(t)\left(2 c_{1}(0, \bar{t})-c_{1}(0, \hat{t}) d t\right. \\
> & 0
\end{aligned}
$$

Therefore, $\rho_{1}$ guarantees provider 1 positive profit for $\sigma=$ $[0, \hat{t}]_{\rightarrow 1}$ for any $\hat{t} \in\left[0, t_{\max }\right]$. 
In words, Theorem 2 says that provider 1 always has a strategy which is individually rational (independent of the other provider's action) and that results in strictly positive profit for all individually rational responses of provider 2 . This is especially attractive if provider 1 could only obtain zero profit as a conservative provider. As we can see from the proof, a possible such pricing strategy is given by $p_{1}^{f}=c_{1}(0, \bar{t})$ and $p_{1}^{\ell}=\frac{c_{1}(0, \bar{t})}{\int_{0}^{t} f(t) t d t}$.

This still leaves the question whether a provider who obtains positive profit as a conservative provider should also become innovative. As the following proposition shows, the answer is usually yes.

Theorem 3. Let provider 2 be conservative, i.e., $p_{2}^{\ell}=0$. If provider 1 obtains strictly positive profit in some constant $B N E$ then there exists a strategy $\rho_{1}$ with $p_{1}^{\ell}>0$ that, for any individually rational response of provider 2 , guarantees provider 1 greater profit than in any constant BNE.

Proof. Recall that by Proposition 1, if provider 1 has positive profit in some constant equilibrium then

$$
c_{1}\left(0, t_{\max }\right)<c_{2}\left(0, t_{\max }\right) .
$$

Let $\bar{t}$ be a type such that

$$
\bar{t}=\operatorname{argmin}_{t} \frac{c_{2}\left(t, t_{\max }\right)-c_{2}\left(0, t_{\max }\right)}{t} .
$$

For any $\epsilon>0$ with $\epsilon<\mu\left(0, t_{\max }\right) \frac{\left.c_{2}\left(\bar{t}, t_{\max }\right)-c_{2}\left(0, t_{\max }\right)\right)}{\bar{t}}$, let $\rho_{1}=\left(p_{1}^{f}, p_{1}^{\ell}\right)$ with $p_{1}^{f}=c_{2}\left(0, t_{\max }\right)-\epsilon$ and $p_{1}^{\ell}=$ $\frac{c_{2}\left(\bar{t}, t_{\max }\right)-c_{2}\left(0, t_{\max }\right)}{\bar{t}}$. Then for any $p_{2}^{f}$ provider 2 obtains no users or there exists a $t>\hat{t}$ such that he obtains all users with type $t>\hat{t}$ and his profit is given by

$$
\begin{aligned}
& \pi_{2}\left(\rho_{1}, \rho_{2}, \sigma\right)=\int_{\hat{t}}^{t_{\max }} f(t)\left(p_{2}^{f}-c_{2}\left(\hat{t}, t_{\text {max }}\right)\right) d t \\
= & \int_{\hat{t}}^{t_{\max }} f(t)\left(p_{1}^{f}+\hat{t} p_{1}^{\ell}-c_{2}\left(\hat{t}, t_{\max }\right)\right) d t \\
\leq & \int_{\hat{t}}^{t_{\max }} f(t)\left(p_{1}^{f}+\hat{t} \frac{c_{2}\left(\hat{t}, t_{\max }\right)-p_{1}^{f}+\epsilon}{\hat{t}}\right. \\
& \left.-c_{2}\left(\hat{t}, t_{\max }\right)\right) d t \\
= & 0-\left(F\left(t_{\max }\right)-F(\hat{t})\right) \epsilon
\end{aligned}
$$

Therefore, provider 2 has no individually rational response for which he obtains any users. It follows that for any individually rational response of provider 2 and any $\epsilon<$ $\mu\left(0, t_{\max }\right) \frac{c_{2}\left(\bar{t}, t_{\max }\right)-c_{2}\left(0, t_{\max }\right)}{\bar{t}}$ provider 1 's profit is

$$
\begin{aligned}
& \pi_{1}\left(\rho_{1}, \rho_{2}, \sigma\right) \\
= & \int_{0}^{t_{\max }} f(t)\left(p_{1}^{f}+t p_{1}^{\ell}-c_{1}\left(0, t_{\max }\right)\right) d t \\
= & c_{2}\left(0, t_{\max }\right)-c_{1}\left(0, t_{\max }\right)-\epsilon \\
& +\mu\left(0, t_{\max }\right) \frac{\left.c_{2}\left(\bar{t}, t_{\max }\right)\right)-c_{2}\left(0, t_{\max }\right)}{\bar{t}} \\
> & c_{2}(0, \hat{t})-c_{1}(0, \hat{t})
\end{aligned}
$$

By Proposition 1, the profit therefore is greater than the profit in any constant BNE.
Note that, in contrast to the strategies described by the proof of Theorem 2, the strategies described by the proof of Theorem 3 are not guaranteed to be individually rational responses to every possible action provider 2 might play. However, they are guaranteed to be individually rational responses to every constant BNE strategy of provider 2.

\subsection{Both Providers are Innovative}

Once one provider starts to employ linear pricing, the other provider might at some point also want to follow. Consequently, we now look at the case where both providers are innovative. When both providers employ linear pricing, the first provider loses much of the additional power he had when the other provider stayed conservative. Consequently, there is no general guarantee that he can still improve his profit. But as long as the cost functions are strictly split-convex, he can still choose a strategy which guarantees him that profits do not decrease compared to any constant BNE (for any individually rational response of the other provider).

Theorem 4. Assume the cost function of provider 2 is strictly split-convex. Then there exists a strategy $\rho_{1}$ with $p_{1}^{\ell}>0$ that, for any individually rational response of provider 2 , guarantees provider 1 greater or equal profit than in any constant $B N E$.

Proof. If provider 1 obtains 0 profit in all constant BNEs, nothing has to be shown. Otherwise, assume provider 1 plays $\rho_{1}=\left(p_{1}^{f}, p_{1}^{\ell}\right)$ with $p_{1}^{\ell}=\left.\frac{d}{d t}\right|_{t_{\max }} c_{2}(0, t)$ and $p_{1}^{f}+$ $p_{1}^{\ell} \mu\left(0, t_{\max }\right)=c_{2}\left(0, t_{\max }\right)$. By Proposition 2 , we know that for all $\rho_{2}$ with utility-maximizing $\sigma=[0, \hat{t}]_{\rightarrow 2}$ it holds that $\pi_{2}\left(\rho_{1}, \rho_{2}, \sigma\right) \leq \pi_{2}\left(\rho_{1}, \rho_{1}, \sigma\right)$ and from strict split-convexity it follows that

$$
\begin{aligned}
& c_{2}(0, \hat{t}) \\
> & c_{2}\left(0, t_{\max }\right) \\
& +\left.\frac{d}{d t}\right|_{t_{\max }} c_{2}(0, t)\left(\mu(0, \hat{t})-\mu\left(0, t_{\max }\right)\right) \\
= & p_{1}^{f}+p_{1}^{\ell} \mu\left(0, t_{\max }\right)+p_{1}^{\ell}\left(\mu(0, \hat{t})-\mu\left(0, t_{\max }\right)\right) \\
= & p_{1}^{f}+p_{1}^{\ell} \mu(0, \hat{t})
\end{aligned}
$$

and therefore

$$
\begin{aligned}
& \pi_{2}\left(\rho_{1}, \rho_{1}, \sigma\right)=\int_{0}^{\hat{t}} f(t)\left(p_{1}^{f}+t p_{1}^{\ell}-c_{2}(0, \hat{t})\right) d t \\
< & \int_{0}^{\hat{t}} f(t)\left(p_{1}^{f}+t p_{1}^{\ell}-p_{1}^{f}-p_{1}^{\ell} \mu(0, \hat{t})\right) d t \\
= & \int_{0}^{\hat{t}} f(t)\left(t p_{1}^{\ell}-p_{1}^{\ell} \mu(0, \hat{t})\right) d t \\
= & 0 .
\end{aligned}
$$

The proof works analogously for $\sigma=[0, \hat{t}]_{\rightarrow 1}$. Therefore, any individually rational response of provider 2 results in user strategy profile $\sigma=\left[0, t_{\max }\right] \rightarrow 1$. For this strategy profile, provider 1 has profit $\pi_{1}\left(\rho_{1}, \rho_{2}, \sigma\right)=c_{1}\left(0, t_{\max }\right)-$ $c_{2}\left(0, t_{\max }\right)$, which by Proposition 1 is the highest possible profit for any constant BNE. 
On the other hand, if both providers are symmetric and their costs are split-convex, moving to linear prices cannot lead to any profit in equilibrium.

Proposition 3. Assume providers are symmetric, i.e., $c_{1}(\cdot)=$ $c_{2}(\cdot)$, and costs are split-convex. Then there can be no BNE with strictly positive profit for either provider.

Proof. If, w.l.o.g., all users prefer provider 1 and he obtains strictly positive payoff, provider 2 obtains zero profit but can deviate to $p_{2}^{f}=p_{1}^{f}-\epsilon, p_{2}^{\ell}=p_{1}^{\ell}$ to obtain strictly positive payoff for $\epsilon>0$ small enough. Therefore, in any potential $\mathrm{BNE}$, the market is split between providers or both obtain zero profit. Assume $\left(\rho_{1}, \rho_{2}, \sigma\right)$ is a BNE and w.l.o.g. $\sigma=$ $[0, \hat{t}]_{\rightarrow 1}$ for some $0<\hat{t}<t_{\max }$. By Corollary 1 we can assume $\rho_{1}=\rho_{2}$. Then, for any $\epsilon>0$ and $\hat{\rho}_{2}=\left(p_{2}^{f}-\epsilon, p_{2}^{\ell}\right)$ all users prefer provider 2 , resulting in profit

$$
\begin{aligned}
& \pi_{2}\left(\rho_{1}, \hat{\rho}_{2},\left[0, t_{\max }\right]_{\rightarrow 2}\right) \\
= & \int_{0}^{t_{\max }} f(t)\left(p_{2}^{f}-\epsilon+t p_{2}^{\ell}-c_{2}\left(0, t_{\max }\right)\right) d t \\
= & \int_{0}^{t_{\max }} f(t)\left(p_{2}^{f}+t p_{2}^{\ell}\right) d t-\epsilon-c_{2}\left(0, t_{\max }\right) \\
\geq & \int_{0}^{t_{\max }} f(t)\left(p_{2}^{f}+t p_{2}^{\ell}\right) d t-\epsilon \\
& -(1-F(\hat{t})) c_{2}\left(\hat{t}, t_{\max }\right)+F(\hat{t}) c_{2}(0, \hat{t}) \\
= & \pi_{2}\left(\rho_{1}, \rho_{2}, \sigma\right)+\pi_{1}\left(\rho_{1}, \rho_{2}, \sigma\right)-\epsilon
\end{aligned}
$$

If $\pi_{2}\left(\rho_{1}, \rho_{2}, \sigma\right)>0$ or $\pi_{1}\left(\rho_{1}, \rho_{2}, \sigma\right)>0$, it follows that for $\epsilon$ small enough, $\pi_{2}\left(\rho_{1}, \hat{\rho}_{2}, \hat{\sigma}\right)>\pi_{2}\left(\rho_{1}, \rho_{2}, \sigma\right)$, contradicting our assumption that $\left(\rho_{1}, \rho_{2}, \sigma\right)$ is a BNE. Thus, it must hold that $\pi_{2}\left(\rho_{1}, \rho_{2}, \sigma\right)=0$ and $\pi_{1}\left(\rho_{1}, \rho_{2}, \sigma\right)=0$.

When cost functions are not split-convex, symmetric providers still always obtain the same profit in any BNE, even though it can be positive.

Proposition 4. Assume providers are symmetric, i.e., $c_{1}(\cdot)=$ $c_{2}(\cdot)$. Then, in all BNEs it holds that $\pi_{1}\left(\rho_{1}, \rho_{2}, \sigma\right)=$ $\pi_{2}\left(\rho_{1}, \rho_{2}, \sigma\right)$.

Proof. To see this, note that for symmetric providers, whoever has lower profits could switch users with the other one by making an infinitesimal change to his price function.

By definition, whether a tuple $\left(\rho_{1}, \rho_{2}, \sigma\right)$ is a BNE is decided via a three-dimensional condition space, as the profit has to be better than the profit for any other tuple $\left(\hat{\rho_{1}}, \hat{\rho_{2}}, \hat{\sigma}\right)$. This makes it very hard to evaluate whether a given tuple is a BNE. The following theorem instead characterizes equilibria by a one-dimensional condition space, greatly reducing the complexity of checking candidate equilibria.

Theorem 5. A tuple $\left(\rho_{1}, \rho_{2},[0, \hat{t}]_{\rightarrow i}\right)$ is a BNE if and only if $\rho_{1}=\rho_{2}$ and

$$
\begin{aligned}
& F(\hat{t}) c_{i}(0, \hat{t})-F(a) c_{i}(0, a) \\
\leq & \left(F(\hat{t})-F(a)\left(p_{i}^{f}+\mu(a, t) p_{i}^{\ell}\right)\right. \\
\leq & (1-F(a)) c_{-i}\left(a, t_{\max }\right)-(1-F(\hat{t})) c_{-i}\left(\hat{t}, t_{\max }\right)
\end{aligned}
$$

for all $0 \leq a \leq t_{\max }$. If the providers are not symmetric, it also has to hold for all $0 \leq a \leq t_{\max }$ that

$$
\begin{aligned}
& F(a)\left(p_{i}^{f}+\mu(0, a) p_{i}^{\ell}-c_{-i}(0, a)\right) \\
\leq & (1-F(\hat{t}))\left(p_{i}^{f}+\mu\left(\hat{t}, t_{\max }\right) p_{i}^{\ell}-c_{-i}\left(\hat{t}, t_{\max }\right)\right)
\end{aligned}
$$

and

$$
\begin{aligned}
& (1-F(a))\left(p_{i}^{f}+\mu\left(a, t_{\max }\right) p_{i}^{\ell}-c_{i}\left(a, t_{\max }\right)\right) \\
\leq & F(\hat{t})\left(p_{i}^{f}+\mu(0, \hat{t}) p_{i}^{\ell}-c_{i}(0, \hat{t})\right) .
\end{aligned}
$$

The proof is provided in Appendix A.

While Theorem 5 fully characterizes all BNEs, it is a very technical characterization. It can be used to check whether a given tuple $\left(\rho_{1}, \rho_{2}, \sigma\right)$ is a BNE, but it does not enable an easy search procedure for finding candidate BNEs. This is particularly important because a $\left(\rho_{1}, \rho_{2},[0, \hat{t}]_{\rightarrow i}\right)$ that satisfies the conditions of Theorem 5 does not always exist. Thus, even if both providers are innovative, there are cases where no BNE exists. The following corollary addresses this issue, identifying a small subset of user strategy profiles that can be part of a BNE and reducing the search for corresponding provider strategies to a one-dimensional search.

Corollary 2. If a tuple $\left(\rho_{1}, \rho_{2}, \sigma\right)$ with $0<\hat{t}<t_{\max }$ is a $B N E$ and the cost functions are differentiable, then it holds

$$
\begin{aligned}
& \left.\frac{d}{d t}\right|_{\hat{t}} F(t) c_{2}(0, t) \\
= & f(\hat{t})\left(p^{f}+\hat{t} p^{\ell}\right) . \\
= & \left.\frac{d}{d t}\right|_{\hat{t}}-(1-F(t)) c_{1}\left(t, t_{\max }\right)
\end{aligned}
$$

Proof. Note that for $t=\hat{t}$ it trivially holds

$$
\begin{aligned}
& F(\hat{t}) c_{2}(0, \hat{t})-F(t) c_{2}(0, t) \\
= & (F(\hat{t}))-F(t)\left(p_{1}^{f}+\mu(t, t) p_{1}^{\ell}\right) \\
= & (1-F(t)) c_{1}\left(t, t_{\max }\right)-(1-F(\hat{t})) c_{1}\left(\hat{t}, t_{\max }\right) .
\end{aligned}
$$

Theorem 5 further gives us that the three expressions have the same ordering for all $t$, especially for all $t$ in any small neighborhood around $\hat{t}$. Therefore, all three expressions need to have the same derivative in $t$ at point $t=\hat{t}$.

Given a cutoff point $\hat{t}$, all potential BNE price functions lie on a line defined by Equation (63). To find a BNE, all that remains to be done is to check whether $\hat{t}$ with any of the $\left(p^{f}, p^{\ell}\right)$ on that line satisfy the conditions of Theorem 5.

\section{Welfare Analysis}

In this section, we analyze the impact of variance-based pricing on social welfare. In our model, the social welfare is simply the negative sum of the expected costs of both providers, i.e., $w\left(\rho_{1}, \rho_{2},[0, t]_{i}\right)=-F(\hat{t}) c_{i}(0, \hat{t})-(1-$ $F(\hat{t})) c_{-i}\left(\hat{t}, t_{\max }\right)$. The social welfare in any constant BNE then follows directly from Proposition 1.

Corollary 3. Let both providers be conservative and w.l.o.g. assume $c_{1}\left(0, t_{\max }\right) \leq c_{2}\left(0, t_{\max }\right)$. Then the social welfare in any $B N E$ is $w\left(\rho_{1}, \rho_{2}, \sigma\right)=-c_{1}\left(0, t_{\max }\right)$. 
Proof. Follows directly from Proposition 1.

If only one provider is innovative, the social welfare often goes down, as the innovative provider can employ his additional market power to force the conservative provider to give up part of the market, even if it increases overall costs. But if both are innovative, he loses this power and in BNE, the social welfare cannot decrease compared to any constant BNE.

Proposition 5. W.l.o.g. assume $c_{1}\left(0, t_{\max }\right) \leq c_{2}\left(0, t_{\max }\right)$. When both providers are innovative, then the social welfare in any $B N E\left(\rho_{1}, \rho_{2}, \sigma\right)$ is higher than the social welfare in any constant $B N E$, i.e., $w\left(\rho_{1}, \rho_{2}, \sigma\right) \geq-c_{1}\left(0, t_{\max }\right)$.

Proof. Recall that the social welfare is $w\left(\rho_{1}, \rho_{2} \cdot[0, t]_{\rightarrow i}\right)=$ $(-1)\left(F(\hat{t}) c_{i}(0, \hat{t})+(1-F(\hat{t})) c_{-i}\left(\hat{t}, t_{\max }\right)\right)$. Thus, whenever all users choose provider 1 , the social welfare is the same as in any constant BNE, i.e., $w\left(\rho_{1}, \rho_{2},\left[0, t_{\max }\right]_{\rightarrow 1}\right)=$ $-c_{1}\left(0, t_{\max }\right)$.

We now show the claim of the proposition by contradiction. Assume $\left(\rho_{1}, \rho_{2},[0, \hat{t}]_{\rightarrow i}\right)$ for $i \in 1,2$ is an innovative $\mathrm{BNE}$ where the social welfare is strictly lower than the social welfare in any constant BNE. Then the social welfare under $\left(\rho_{1}, \rho_{2},[0, \hat{t}]_{\rightarrow i}\right)$ is also strictly lower than under $\left(\rho_{1}, \rho_{2},\left[0, t_{\max }\right] \rightarrow 1\right)$, i.e., the sum of the expected costs is strictly higher; formally: $F(\hat{t}) c_{i}(0, \hat{t})+(1-$ $F(\hat{t})) c_{-i}\left(\hat{t}, t_{\max }\right)>c_{1}\left(0, t_{\max }\right)$. Additionally, by Corollary 1 , we can assume that $\rho_{1}=\rho_{2}$. This means that the payment of any user is independent of which provider he chooses, and therefore the sum of the revenues of both providers does not change between $\left(\rho_{1}, \rho_{2},[0, \hat{t}]_{\rightarrow i}\right)$ and $\left(\rho_{1}, \rho_{2},[0, \hat{t}]_{\rightarrow 1}\right)$. Taken together, when going from the first profile to the second profile, the sum of the revenues stay the same but the costs strictly decrease, which implies that $\pi_{1}\left(\rho_{1}, \rho_{2},\left[0, t_{\max }\right]_{\rightarrow 1}\right)>\pi_{1}\left(\rho_{1}, \rho_{2},[0, \hat{t}]_{\rightarrow i}\right)+$ $\pi_{2}\left(\rho_{1}, \rho_{2},[0, \hat{t}]_{\rightarrow i}\right)$. Therefore, $\left(\rho_{1}, \rho_{2},[0, \hat{t}]_{\rightarrow i}\right)$ can not be a BNE, a contradiction.

\section{Numerical Example}

In this section, we illustrate our results via a simple numerical example. We assume that user types are uniformly distributed on $[0,1]$. Further, we assume provider 1 has cost function $c_{1}(a, b)=0.0125+\mu(a, b)^{2}$ and provider 2 has cost function $c_{2}(a, b)=0.2+\frac{\mu(a, b)^{2}}{4}$, where $\mu(a, b)$ denotes the average type of all users in $[a, b]$ (as previously defined in Section 2.1). Thus, provider 1 has a lower cost for low types but a higher cost for high types than provider 2, and both providers have the same cost for the whole user population. From Proposition 1, we know that when both providers are conservative, there are only zero-profit BNEs. They occur at $p_{1}^{f}=p_{2}^{f}=0.2625$ with a welfare of -0.2625 .

We now consider each provider unilaterally switching to linear prices (as described in Theorem 2). Figure 1 shows the profit increase that provider 1 could obtain when unilaterally innovating by playing $\left(p_{1}^{f}=0.215, p_{1}^{\ell}=0.5309\right)$ while provider 2 remains conservative. Figure 2 shows the analogous result for when provider 2 becomes innovative. We see that provider 1 innovating leads to the overall better result

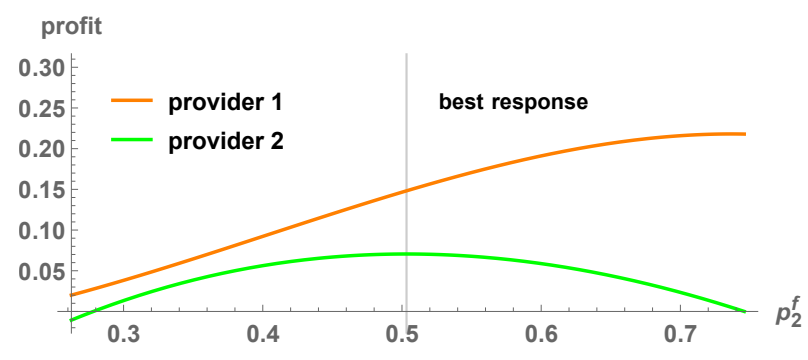

Figure 1: Profit of both providers for all conservative responses of provider 2 to provider 1 playing $\left(p_{1}^{f}=0.215, p_{1}^{\ell}=0.5309\right)$.

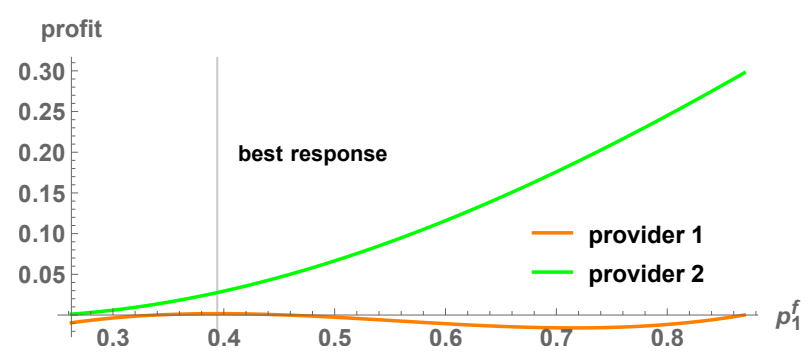

Figure 2: Profit of both providers for all conservative responses of provider 1 to provider 2 playing $\left(p_{2}^{f}=0.2506, p_{2}^{\ell}=0.6188\right)$.

for both providers. This is not surprising, considering that the innovative provider obtains the lower type portion of the market in which provider 1 has lower costs. At the best response of provider 2 , the social welfare therefore increases to -0.2077 . Nonetheless, if provider 2 innovates instead, he can still obtain a profit of 0.0442 at provider 1 's best constant response of $p_{1}^{f}=0.3941$. Since here, provider 2 uses the power of his larger strategy space as an innovative provider to force provider 1 to obtain a high type population interval (for which provider 1 has higher costs) social welfare unsurprisingly decreases to -0.3846 .

For the case where both providers are willing to employ linear pricing, Corollary 2 provides us with conditions on candidate equilibrium user strategy profiles. For our example, we can use those conditions to find four cutoff points: $\sigma=[0,0.595]_{\rightarrow 1}, \sigma=[0.5431,1]_{\rightarrow 1}, \sigma=[0,1]_{\rightarrow 1}$ and $\sigma=[0,0]_{\rightarrow 1}$. All of these except for $\sigma=[0,0.595]_{\rightarrow 1}$ do not satisfy Theorem 5 and are eliminated. For $\sigma=[0,0.595] \rightarrow 1$ any $p_{1}^{f}=p_{2}^{f} \in[0,0.0409], p_{1}^{\ell}=p_{2}^{\ell}=\frac{0.2784-p_{1}^{f}}{0.595}$ satisfy Theorem 5 and form equilibrium pricing strategies. To visualize an example BNE, Figure 3 shows the profit of both providers for $p_{1}^{f}=p_{2}^{f}=0$ and $p_{1}^{\ell}=p_{2}^{\ell}=0.4676$ for different utility-maximizing $\sigma=[0, t]_{\rightarrow 1}$. We see that neither provider wants to deviate to enforce a different user strategy profile $\sigma=[0, t]_{\rightarrow 1}$ than $\sigma=[0,0.595]_{\rightarrow 1}$. Social welfare at this BNE is -0.2055 , which is even slightly better than when only provider 1 was innovative. However, the increased competition leads to a significantly lower profit for both providers than when only provider 1 was innovative. This suggests that a conservative provider who is forward-looking and properly anticipates the possible BNEs may prefer to stay conservative if the other provider is already innovative. 


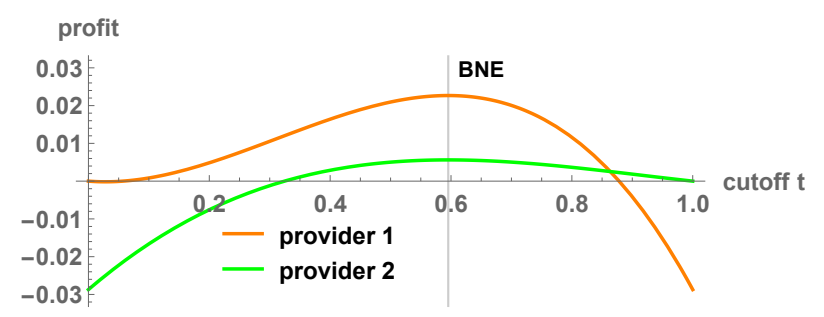

Figure 3: Profit of both providers for different $\sigma=[0, t]_{\rightarrow 1}$

\section{Conclusion}

In this paper, we have studied the competitive effects of providers utilizing linear variance-based (or type-based) pricing rules in settings where a provider's costs depend on the average type of all his users. We have shown that, while a single provider innovating often leads to non-existence of BNEs, the innovative provider can exert the additional market power he has due to his larger strategy space to unilaterally set prices that increase his profit for all individually rational responses of a conservative provider. We have further characterized all equilibria where both providers employ linear pricing. While much of the additional market power of an innovative provider is lost once the other provider also adopts linear pricing, the increased strategy space allows providers to split the market more closely along differences in their cost functions. This often increases both providers' profits and social welfare. While our general insights translate to settings with more than two providers, the strategic considerations and the BNEs quickly become too complex to provide useful insights. In future work, it would be interesting to study secondary effects like incentivizing users to actively lower their variance. Furthermore, to deploy variance-based pricing in practice, it would be important to develop good machine learning algorithms to effectively learn the users' types over time.

\section{A Proof of Theorem 5}

Proof. A tuple $\left(\rho_{1}, \rho_{2},[0, \hat{t}]_{\rightarrow i}\right)$ is a BNE if $[0, \hat{t}]_{\rightarrow i}$ is utility maximizing and no provider has a profitable deviation. From Proposition 2 we know that any deviation with different prices is worse than keeping the same prices and choosing the deviating provider's most-preferred utility-maximizing user strategy profile. Thus, the tuple is a BNE if and only if moving to any different user strategy profile (weakly) decreases both providers' profits. W.l.o.g. assume $i=2$, and thus, in the candidate BNE we consider, provider 2 obtains the lowvariance users. We first consider how profits change under different user strategy profiles for which provider 2 still obtains the low-variance users. Changing to any $[0, a]_{\rightarrow 2}$ with $0 \leq a<\hat{t}$ yields a profit change for Provider 1 of

$$
\begin{aligned}
& (1-F(\hat{t}))\left(c_{1}\left(\hat{t}, t_{\max }\right)-c_{1}\left(a, t_{\max }\right)\right. \\
& +\int_{a}^{\hat{t}} f(t)\left(p_{1}^{f}+t p_{1}^{\ell}-c_{1}\left(a, t_{\max }\right)\right) d t \\
= & (1-F(\hat{t})) c_{1}\left(\hat{t}, t_{\max }\right)-(1-F(a)) c_{1}\left(a, t_{\max }\right) \\
& +(F(\hat{t})-F(a))\left(p_{1}^{f}+\mu(a, t) p_{1}^{\ell}\right)
\end{aligned}
$$

while with $\hat{t}<a<t_{\max }$ it yields a change of

$$
\begin{aligned}
& (1-F(a))\left(c_{1}\left(\hat{t}, t_{\max }\right)-c_{1}\left(a, t_{\max }\right)\right. \\
& -\int_{\hat{t}}^{a} f(t)\left(p_{1}^{f}+t p_{1}^{\ell}-c_{1}\left(\hat{t}, t_{\max }\right)\right) d t \\
= & (1-F(\hat{t})) c_{1}\left(\hat{t}, t_{\max }\right) \\
& -(1-F(a)) c_{1}\left(a, t_{\max }\right) \\
& +(F(\hat{t})-F(a))\left(p_{1}^{f}+\mu(a, t) p_{1}^{\ell}\right) .
\end{aligned}
$$

Similarly, the profit change for provider 2 for $a<\hat{t}$ is

$$
\begin{aligned}
& F(a)\left(c_{2}(0, \hat{t})-c_{2}(0, a)\right) \\
& -\int_{a}^{\hat{t}} f(t)\left(p_{1}^{f}+t p_{1}^{\ell}-c_{2}(0, \hat{t})\right) d t \\
= & F(\hat{t}) c_{2}(0, \hat{t})-F(a) c_{2}(0, a) \\
& -(F(\hat{t})-F(a))\left(p_{1}^{f}+\mu(a, t) p_{1}^{\ell}\right)
\end{aligned}
$$

and for $a>\hat{t}$ the profit change is given by

$$
\begin{aligned}
& F(\hat{t})\left(c_{2}(0, \hat{t})-c_{2}(0, a)\right) \\
& +\int_{\hat{t}}^{a} f(t)\left(p_{1}^{f}+t p_{1}^{\ell} c_{2}(0, a)\right) d t \\
= & F(\hat{t}) c_{2}(0, \hat{t})-F(a) c_{2}(0, a) \\
& -(F(\hat{t})-F(a))\left(p_{1}^{f}+\mu(a, t) p_{1}^{\ell}\right) .
\end{aligned}
$$

Bounding all of these expressions above by zero yields the first half of the theorem.

Equivalently, we now consider how the profit changes when provider 1 obtains the low variance users (i.e. $[0, a]_{\rightarrow 1}$ ):

$$
\begin{aligned}
& \int_{0}^{a} f(t)\left(p_{1}^{f}+t p_{1}^{\ell}-c_{1}(0, a)\right) d t \\
& -\int_{\hat{t}}^{t_{\max }} f(t)\left(p_{1}^{f}+t p_{1}^{\ell}-c_{1}\left(\hat{t}, t_{\max }\right)\right) d t \\
= & F(a)\left(p_{1}^{f}+\mu(0, a) p_{1}^{\ell}-c_{1}(0, a)\right) \\
& -(1-F(\hat{t}))\left(p_{1}^{f}+\mu\left(\hat{t}, t_{\text {max }}\right) p_{1}^{\ell}-c_{1}\left(\hat{t}, t_{\text {max }}\right)\right) \\
\leq & 0
\end{aligned}
$$

and

$$
\begin{aligned}
& \int_{a}^{t_{\max }} f(t)\left(p_{1}^{f}+t p_{1}^{\ell}-c_{2}\left(a, t_{\max }\right)\right) d t \\
& -\int_{0}^{\hat{t}} f(t)\left(p_{1}^{f}+t p_{1}^{\ell}-c_{2}(0, \hat{t})\right) d t \\
= & (1-F(a))\left(p_{1}^{f}+\mu\left(a, t_{\max }\right) p_{1}^{\ell}-c_{2}\left(a, t_{\max }\right)\right) \\
& -F(\hat{t})\left(p_{1}^{f}+\mu(0, \hat{t}) p_{1}^{\ell}-c_{2}(0, \hat{t})\right) \\
\leq & 0
\end{aligned}
$$

If the providers are symmetric, both providers would get the same profit if they served the same segment of the market, and therefore the conditions $(79)-(81)$ and $(84)-(86)$ do not have to be checked. 


\section{References}

[Baye and Kovenock, 2017] Michael R. Baye and Dan Kovenock. Bertrand Competition, pages 1-7. Palgrave Macmillan UK, London, 2017.

[Blattberg and Wisniewski, 1989] Robert C Blattberg and Kenneth J Wisniewski. Price-induced patterns of competition. Marketing Science, 8(4):291-309, 1989.

[Celebi and Fuller, 2012] Emre Celebi and J David Fuller. Time-of-use pricing in electricity markets under different market structures. IEEE Transactions on Power Systems, 27(3):1170-1181, 2012.

[Cramton, 2017] Peter Cramton. Electricity market design. Oxford Review of Economic Policy, 33(4):589-612, 2017.

[Dierks and Seuken, 2019] Ludwig Dierks and Sven Seuken. Cloud pricing: The spot market strikes back. In Proceedings of the 2019 ACM Conference on Economics and Computation. ACM, 2019.

[Dierks et al., 2019] Ludwig Dierks, Ian A. Kash, and Sven Seuken. On the cluster admission problem for cloud computing. In Proceedings of the 14th Workshop on the Economics of Networks, Systems and Computation, June 2019.

[Ericsson, 2017] Ericsson. Shifting mobile data plans, 2017.

[Feng et al., 2013] Yuan Feng, Baochun Li, and Bo Li. Price competition in an oligopoly market with multiple iaas cloud providers. IEEE Transactions on Computers, 63(1):59-73, 2013.

[Gallego et al., 2006] Guillermo Gallego, Woonghee Tim Huh, Wanmo Kang, and Robert Phillips. Price competition with the attraction demand model: Existence of unique equilibrium and its stability. Manufacturing \& Service Operations Management, 8(4):359-375, 2006.

[Joskow and Wolfram, 2012] Paul L Joskow and Catherine D Wolfram. Dynamic pricing of electricity. American Economic Review, 102(3):381-85, 2012.

[López-Pérez et al., 2009] David López-Pérez, Alvaro Valcarce, Guillaume De La Roche, and Jie Zhang. Ofdma femtocells: A roadmap on interference avoidance. IEEE Communications Magazine, 47(9):41-48, 2009.

[Moorthy, 1984] K Sridhar Moorthy. Market segmentation, self-selection, and product line design. Marketing Science, 3(4):288-307, 1984.

[Muratori and Rizzoni, 2015] Matteo Muratori and Giorgio Rizzoni. Residential demand response: Dynamic energy management and time-varying electricity pricing. IEEE Transactions on Power systems, 31(2):1108-1117, 2015.

[Mussa and Rosen, 1978] Michael Mussa and Sherwin Rosen. Monopoly and product quality. Journal of Economic Theory, 18(2):301 - 317, 1978.

[Rong et al., 2018] Jiang Rong, Tao Qin, and Bo An. Dynamic pricing for reusable resources in competitive market with stochastic demand. In Thirty-Second AAAI Conference on Artificial Intelligence, 2018.
[Truong-Huu and Tham, 2014] Tram Truong-Huu and Chen-Khong Tham. A novel model for competition and cooperation among cloud providers. IEEE Transactions on Cloud Computing, 2(3):251-265, 2014.

[Urieli and Stone, 2016] Daniel Urieli and Peter Stone. Autonomous electricity trading using time-of-use tariffs in a competitive market. In Thirtieth AAAI Conference on Artificial Intelligence, 2016.

[Varian, 1989] Hal R Varian. Price discrimination. Handbook of Industrial Organization, 1:597-654, 1989. 